\title{
TOPOGRAPHIE DES NOUVEAUX LIEUX DE CONFLICTUALITÉ POLITIQUE
}

\author{
Nicolas Baygert ${ }^{1}$
}

Avec l'avènement du Web 2.0, les « marques politiques » disposent de nouveaux outils pour cibler et mobiliser leur électorat. Mais au lieu d'accompagner une mouvance « bottom-up », favorisée par l'émergence des médias sociaux, les marques politiques doivent faire face à de nouveaux lieux de jugement collectifs et interactifs. Dans un dialogue en temps réel où le citoyenconsommateur devient partie prenante, on assiste à l'émergence de nouveaux mouvements citoyens 100\% grassroots: une contre-opinion cyber-diffusée cherchant à influer sur le débat publique et à donner le La dans cette « grande conversation ». Une agora virtuelle qui s'érige progressivement en panoptique participatif, où il s'agira désormais de soigner sa « réputation digitale », soumise au jugement constant exercé par les citoyensconsommateurs.

La percée de dispositifs de communication interactifs et personnalisés - les médias sociaux tels Facebook ou Twitter-suggère aujourd'hui un rapport davantage horizontal entre des citoyen-consommateurs de

1 Nicolas Baygert est doctorant à l'Ecole de communication de l'UCL et au CELSA (Paris IV-Sorbonne)..

Recherches en communication, $\mathrm{n}^{\circ} 37$ (2012). 
plus en plus critiques et les mandataires. Un décloisonnement numérique des espaces citoyens débouchant sur une nouvelle dynamique de jugement du politique.

Cette évolution se rapporterait à la notion de « consumérisme politique », qui induit de la part d'individus émancipés des carcans de l'engagement politique traditionnel, un besoin de juger les «marques politiques » en temps réel. Les individus étant à même d'exercer un réel pouvoir de vigilance et de dénonciation vis-à-vis du politique à travers les médias sociaux, cette dynamique de jugement devra dorénavant être prise en compte par les partis. Aussi, cet article veillera à illustrer comment cette évolution est susceptible d'affecter un champ où l'on décèle des logiques de mobilisation alternative, de surveillance, voire de boycott.

Aux États-Unis, la campagne d'Howard Dean en 2003 fut la première à démontrer le potentiel des médias sociaux à s'insérer durablement dans les pratiques politiques d'une proportion importante de la population (Trippi, 2005). La mise en orbite de Ségolène Royal en 2007 et la campagne de Barack Obama l'année suivante ont également dévoilé les prémisses d'un nouveau type de rapport au pouvoir sur base d'un plébiscite virtuel. Mais alors que beaucoup d'espoirs étaient placés dans l'idée d'un renouvèlement du processus électoral à travers Internet (Vedel, 2003), voire l'émergence d'une citoyenneté numérique (Boullier, 2008, p. 83), c'est davantage à une revitalisation de la conflictualité à travers les réseaux sociaux que l'on assiste à présent ; un « espace public oppositionnel » (Negt, 2007) alimentant en continu la " grande conversation » autour des marques politiques et qui chercherait à affecter la prise de décision et le processus de sélection des élites.

Comme caisse de résonnance, voire comme matrice de conflit, Internet participe ainsi à l'autonomisation progressive de l'activisme politique - en périphérie du cadre partisan classique - et à l'émergence d'initiatives militantes spontanées, bottom-up ou collaboratives. Le présent article s'intéressera à plusieurs mouvements autogérés - tels le Tea Party, le Movimento Cinque Stelle et le Parti Pirate - illustrant cette dynamique de mobilisation alternative.

\section{Des marques au consumérisme politique}

La notion de « marque politique »- concept témoignant d'une réification symbolique de la gouvernance - s'inscrit dans une dédifférenciation généralisée du champ de la consommation, qui concernerait 
l'ensemble des rapports sociaux. Bryman (2002) ou encore Lipovetsky (2006, p. 153) évoquent une société « où la forme-consommation apparaît comme le schéma organisateur des activités individuelles, où l'éthos du consumérisme restructure toutes les sphères y compris celles qui sont extérieures à l'échange payant. »

À travers ce prisme de marques, le politique se conforme aux impératifs communicationnels en vigueur au sein d'une société de consommation dédifférenciée, en adoptant une sémantique englobante - d'après une terminologie propre à Niklas Luhmann ${ }^{1}$ (Ferrarese, 2007, p. 39) - la « marque politique » mettant en lumière la convergence du politique et du consommable. La notion de marque correspond à un style, une façon d'incarner sa fonction (Séguéla \& Saussez, 2007, p. 11) et suggère ici un recentrage des débats autour de la personnalité et de la vie privée des candidats; de leur image de marque.

Ce glissement vers les marques aurait favorisé l'avènement d'un « consumérisme politique » selon lequel la souveraineté du consommateur s'impose comme modèle de souveraineté politique - un phénomène déjà répertorié par Tony Blair, il y a deux décennies (Blair, 1992, p. 3). Depuis le milieu des années 1990, les chercheurs ont ainsi analysé la manière dont les décisions des consommateurs influence la politique (Micheletti, 2003, p. 15). Désignant à l'origine les mouvements sociaux soucieux d'une consommation responsable et engagée, le consumérisme politique incarne également l'émancipation du citoyen-consommateur vis-à-vis des obligations symboliques de classes. Le comportement politique dédifférencié ne résulte plus d'un habitus social qui, d'après Pierre Bourdieu (1979) caractérise une " affinité de style », un « air de famille », une "systématicité ». Aussi, comme consommacteur émancipé, le citoyen procède désormais lui-même à la sélection des benchmarks - les indicateurs de performance à partir desquels les marques politiques seront jugées.

Le consumérisme politique symbolise dès lors la manière dont le citoyen-consommateur intervient dans le processus de co-construction de l'offre politique. Selon Bolz (2002), les individus agissent en prosumers : ils coproduisent leur propre consommation en co-construisant la marque et en interagissant sur ses modalités de diffusion ou, inver-

1 Pour Luhmann, tout système social étant constitué de communication, la différenciation entre sous-systèmes dépend de la production de sémantiques autonomes. Le phénomène de dédifférenciation ici traité correspondrait dès lors à la convergence autour d'une sémantique consumériste. 
sement, de boycott. Un boycott qui représenterait le versant négatif du consumérisme politique - un principe selon lequel les citoyensconsommateurs, réprouvent la marque de manière collective.

Avec l'émergence des médias sociaux, les marques politiques entrent de plain-pied dans une conversation critique avec les citoyensconsommateurs, devenus parties prenantes. La démocratie est entrée dans un nouvel âge «dans lequel la définition du bien commun n'est plus du seul ressort des gouvernants et doit désormais se partager et se discuter » (D’Almeida, 2007, p. 52). Le centre de gravité de la démocratie s'étant progressivement déplacé de l'espace politico-médiatique vers une société en conversation (Cardon, 2010), une large proportion du contenu produit par les internautes vise dorénavant à alimenter cette conversation en continu. L'important n'étant pas tant l'élaboration de consensus mais la possibilité, pour un grand nombre d'individus, d'intégrer le débat et d'exprimer leur avis. En conséquence, à travers les médias sociaux le consumérisme politique prend virtuellement corps.

\section{Des réseaux sociaux au service de la marque}

Pour les partis, le comportement en ligne des citoyens-consommateurs est devenu un véritable enjeu, la politique devant désormais gagner son existence dans ce nouvel espace, comme elle l'a fait dans les quartiers, sur le territoire physique (Boullier, 2008, p. 87). Une prise en compte de l'importance du Web qui se reflète parfois jusque dans la dénomination des partis, comme l'indique l'exemple du MoDem ${ }^{1}$. La mise ne ligne, depuis 2007, de plateformes partisanes répond toutefois à des finalités d'ordre différent.

Lancée par Ségolène Royal en marge de l'appareil socialiste au moment de la campagne présidentielle française de 2007, la plateforme Désirs d'Avenir ${ }^{2}$ ambitionnait l'échange de contenu sur un mode horizontal. Misant sur « l'intelligence collective» issue des processus participatifs, la parole devait être donnée aux " citoyens-experts » plutôt qu'aux professionnels de la politique. Dans cette entreprise de marketing participatif, on entraperçut les prémisses d'une dynamique démocratique 2.0 dans laquelle des « experts profanes » se regroupent

1 L'acronyme MoDem du Mouvement démocrate, le parti créé par François Bayrou à la suite des Présidentielles françaises de 2007, aurait été inventé par les jeunes UDF sur les forums Internet.

2 Disponible à : http://www.desirsdavenir.org/ 
pour élaborer et exprimer de nouvelles identités, délaissant leurs porteparole usuels (Blondeau, 2007, p. 29). Rémi Lefebvre (2008) décrit le « Royalisme » comme une tentative de redéfinir symboliquement les relations entre représentants et représentés. Ainsi, Désirs d'Avenir représentait la plaque tournante d'une opération de benchmarking politique inédite avec un consomm-acteur en point de mire. En témoigne l'écriture, chapitre par chapitre, d'un livre-programme interactif basé sur les commentaires d'internautes. Grâce à cette dynamique collaborative et à cette mise en réseau, la marque « Ségolène » pu compter sur une visibilité dont elle ne bénéficiait pas à l'origine. Cette utilisation pionnière de l'Internet politique permit à la candidate « d'affirmer tout à la fois son attachement à la démocratie participative, sa modernité et son originalité »(Vedel, 2006, p. 52) mais qui demeura néanmoins au stade cosmétique. Le « Royalisme », détaché de la machine partisane socialiste, ne permit pas d'aboutir à une réelle structuration de l'action militante. En perpétuelle conversation, la marque «Ségolène » ne dégagea pas, en outre, la cohérence programmatique souhaitée.

En 2008, la campagne de Barack Obama aura également tiré profit de toutes les caractéristiques technologiques du Web 2.01. Mais le site my.barackobama.com $(\mathrm{MyBo})^{2}$, qui permettait aux militants de valoriser leurs contributions à la campagne auprès de leurs réseaux sociaux, fut autant consacré à la diffusion de la marque Obama qu'au dispatching des 8000 groupes de soutien et des 750.000 bénévoles actifs. En canalisant les conversations pour en faire un outil de mobilisation locale, la campagne en ligne réussit à prendre le relai d'une importante opération de terrain (grassroots) menée par des militants qui bénéficiaient d'un pouvoir d'initiative (empowerment) considérable.

À l'instar de $M y B o$, en France, l'UMP et le PS mirent en ligne leurs propres réseaux sociaux au début de l'année 2010. À l'origine, la plateforme de l'UMP Créateurs de Possibles ${ }^{3}$ « projet phare de la stratégie de modernisation du mouvement populaire » tel qu'il fut présenté par Xavier Bertrand ${ }^{4}$, privilégiait 1'aspect participatif et communautaire.

1 Les plateformes 2.0 Facebook, MySpace, YouTube, Flickr, BlackPlanet, Faithbase, Eons, Glee, MiGente, MyBatanga, AsianAve, Twitter, Eventfull, Linkedin, DNC Partybuilder, Digg ont toutes été investies.

2 La plateforme MyBo bénéficia notamment de l'expertise de Chris Hughes, cofondateur de Facebook.

3 Disponible à : http://www.lescreateursdepossibles.com/

4 Ancien secrétaire général de l'UMP. 
Comptant 15.000 inscrits en un an, l'entreprise fut considérée comme un échec par son successeur Jean-François Copé1. Avec la CooPol ${ }^{2}$, le PS souhaitait également numériser l'organisation militante du parti. Aussi, l'objectif ici n'était pas le déclenchement d'une dynamique collaborative mais la simplification du quotidien des militants. Un choix illustrant une évolution dans l'appréhension des médias sociaux par les partis. On observe ainsi la substitution progressive d'un idéal participatif, si difficile à implémenter, par un souci de performance organisationnelle, avec la volonté d'introduire davantage d'horizontalité.

Plutôt que de susciter l'interaction directe des militants avec la marque, les partis optent ainsi pour une rationalisation de l'activisme en ligne, en mettant à disposition des internautes une boîte à outils $2.0^{3}$ et en veillant à interconnecter au mieux les dimensions online et offline de l'action militante. La rupture paradigmatique voulue par Ségolène Royal contraste par conséquent avec ces considérations pragmatiques qui relèguent l'élan participatif au second plan. Une tendance soulignée par Blondiaux (2008, p. 74), « la démocratie participative, telle qu'elle se pratique aujourd'hui assez couramment, s'apparente à une série d'exercices de communication dans lesquels l'enjeu réel est bien plus de signifier l'intention de faire participer que de faire participer réellement ».

\section{L'émancipation numérique des citoyens-consommateurs}

Face aux réticences des structures partisanes à transformer les modalités de la participation, les individus disposent de trois options : l'adhésion (loyalty), la défection (exit) ou la prise de parole (voice) (Hirschmann, 1995). Aussi, la montée actuelle de l'impératif participatif peut être interprété comme une demande croissante de prise de parole de la part de mouvements sociaux mais aussi d'individus isolés revendiquant une autonomie par rapport au système politique institutionnel (Blondiaux, 2008, p. 82). Elle traduit également un contexte technologique particulier. Depuis une dizaine d'années, parallèlement à l'émergence d'un web collaboratif qui ne nécessite plus de connais-

1 Les Créateurs de possibles bientôt fermés par l'UMP, article paru dans L'Express (27 décembre 2010).

2 Disponible à : http://www.lacoopol.fr/

3 On citera comme exemple l'application iPhone $M y B$ o, véritable QG de campagne portatif. Disponible à : $\underline{\text { http://my.barackobama.com/page/content/iphone }}$ 
sances techniques particulières de la part des utilisateurs, le savoir-faire entourant les technologies de communication politique et du militantisme en ligne a largement dépassé l'univers du webmarketing politique professionnel. Cette réappropriation démocratique des outils contribue à l'apparition de nouveaux types de mobilisation et à une émancipation progressive de l'activisme politique, en marge de l'engagement idéologique et permanent que constitue traditionnellement l'adhésion à un parti.

Les développements de l'activisme en ligne correspondent à la consécration d'un consommateur de politique nomade, libéré des structures hiérarchisées et institutionnalisées : un consomm-acteur disposé à des formes de consultation, de mobilisation et de prise de décision spontanés et flexibles ${ }^{1}$, dépassant les procédures de la démocratie délégataire (Blondeau, 2007, p. 29).Un phénomène susceptible d'aboutir à des formes d'activisme davantage structurées et durables que l'on peut comparer aux pratiques collaboratives de crowdsourcing ${ }^{2}$, propres au marketing participatif sur Internet ou à la dynamique Peer-to-Peer - le processus collaboratif et décentralisée à l'œuvre dans les réseaux distribués ${ }^{3}$. D'après Pierre Rosanvallon (2006, p. 73), la toile peut ainsi être perçue comme forme sociale à part entière, en même temps qu'une véritable forme politique. Une forme sociale féconde, puisqu'elle concourt sur des modes inédits à la constitution des communautés.

Mise en conversation sur les réseaux sociaux - et dans l'absence de gate-keepers -, la parole publique n'est plus en mesure d'imposer silence et déférence. Elle sera dorénavant commentée, critiquée, raillée, transformée par un grand nombre de personnes autrefois jugées inaptes ou ignorantes (Cardon, 2010, p. 10). Les inflexions données à la marque

1 Les médias sociaux fournissent des outils particulièrement adaptés à ce nouveau type d'engagement $a d$ hoc, permettant de rejoindre (ou de quitter) une action politique très rapidement ou de débattre depuis chez soi. Sur Facebook, un click suffit pour se désaffilier et quitter la Fan Page d'un candidat. Un même click permettra de suivre les « gazouillis » d'élus sur Twitter.

2 Le terme crowdsourcing, " approvisionnement par la foule », est un néologisme inspiré par le vocable « outsourcing " (externalisation). Les pratiques de crowdsourcing misent sur une collaboration de masse favorisée par les technologies issues du Web 2.0 pour aboutir à des objectifs dans le domaine du savoir où de l'économie.

3 Le principe du P2P obéit à un leadership collectif et implique la constitution de structures changeantes, adaptées aux différentes phases de constitution d'un projet (Bauwens \& Sussan, 2005, p. 199). 
en seront directement affectées, donnant lieu à un feedback critique permanent qui alimente le flux des conversations.

Un tissu effiloché de micro-espaces de débats ne cesse de se nouer, de se défaire et de se déplacer sur le web. Les internautes se saisissent de questions locales ou globales. Ils surveillent, commentent discutent et critiquent mille sujets. [...] Cette dissémination capillaire des discussions crée un réseau souple et étonnamment réactif. En certaines circonstances, il parvient à cristalliser l'attention des internautes pour former un foyer plus solide. (Cardon, 2010, p. 70).

\section{Vers une conflictualité contre-démocratique}

L'agrégation massive d'avis et d'engagements individuels sur Internet participe ainsi au renforcement d'un espace public virtuel autonome, voire à une revitalisation de la conflictualité par la configuration d'un « espace public oppositionnel » où seraient libres de s'exprimer des « subjectivités rebelles» (Negt, 2007). Des manifestations de défiance qui, à terme, font politiquement système (Rosanvallon, 2006, p. 12). Ces formes d'expressivités numériques au sein de l'espace oppositionnel vont d'ores et déjà de la mise en place de watchdog committees 2.0 (comité de vigilance) à l'émergence de mouvements citoyens autogérés, jusqu'aux soulèvements démocratiques numériquement coordonnées en Tunisie et en Égypte de 2011. Dans ces exemples qui témoignent du caractère amplifiant des médias sociaux, l'espace public virtuel peut être appréhendé comme la mise en réseau de phénomènes contestataires isolés.

En Belgique, la manifestation ShameBE $E^{1}$ - qui trouva son origine sur Facebook et Twitter - a rassemblé plus de 30.000 personnes le 23 janvier 2011 à Bruxelles. En parallèle, l'initiative Camping 16², un camping virtuel devant le 16 rue de la Loi - adresse du cabinet du Premier ministre belge - appelait les internautes à se réunir derrière un même slogan: « Pas de gouvernement? Remboursement! », illustrant par-là les sommations d'un consumérisme politique revigoré.

$\mathrm{Au}$ cœur de ces mobilisations synonymes d'empowerment numérique figurent des citoyens-consommateurs désormais parties prenantes

1 Disponible à : $\underline{\mathrm{http}: / / \mathrm{www} .230111 . \mathrm{be} /}$

2 Disponible à : http://camping16.be/ 
d'une « grande conversation » autour des marques politiques. Dans cette dynamique d'engagement, on relèvera d'une part, la nécessité d'une justification continue de la décision politique, d'autre part, la volonté de participer au renouveau politique autant par un input constant quant à la sélection des élites, qu'en favorisant l'émergence de formations politiques autogérées. La caractéristique de ces formations est qu'elles s'organisent autour des trois principes de vigilance, de dénonciation et de notation, constituant ainsi le vecteur le plus visible et le plus structuré de ce que Pierre Rosanvallon nomme la contre-démocratie : « une démocratie permanente de la défiance face à la démocratie épisodique de la légitimité électorale » (2006, p. 15).

\section{Topographie des mobilisations contestataires auto-organisées}

Selon l'historien Paul Nolte (2011, p. 9), l'évolution vers une démocratie participative et protestataire constitue vraisemblablement l'évolution la plus importante de l'histoire de la démocratie de la dernière moitié du $20^{\text {ème }}$ siècle. Aussi, dans le contexte des technologies numériques, l'informalité et l'horizontalité sont d'ores et déjà devenues gage de démocratie et de spontanéité (Mathieu, 2011, p. 38).

\section{L'exemple du Tea Party}

Dans l'histoire récente, l'exemple du Tea Party aux États-Unis illustre l'apparition de nouvelles formes de contestation consolidées grâce aux réseaux sociaux. Radicalement conservateur et ancré sociologiquement dans la classe moyenne, le Tea Party ${ }^{1}$, qui évoque l'un des premiers épisodes de la guerre d'indépendance américaine ${ }^{2}$, représente une « coalition de citoyens et d'organismes préoccupés par la tendance récente d'insouciance fiscale du gouvernement ${ }^{3} »$. Rattaché au camp Républicain, il s'oppose dès lors à Washington et à la machine d'État. Le mouvement se perçoit également comme lieu de contradiction consacré à la décrédibilisation de l'image de marque d'Obama — un mouvement d'opposition ad hominem ${ }^{4}$.

1 Disponible à : http://teapartypatriots.ning.com/

2 Son nom s'inspire du "Boston Tea Party », symbolisant la révolte contre le colonisateur anglais au XVIIIème siècle.

3 «A coalition of citizens and organizations concerned about the recent trend of fiscal recklessness in government».

4 De nombreuses critiques ciblèrent Sarah Palin, alors égérie du Tea Party, à la suite de 
De par une dynamique bottom-up le Tea Party inscrit le militantisme dans un projet résolument participatif, renouant ainsi avec la tradition américaine des Town meetings ${ }^{1}$. Le mouvement s'appuie sur des structures locales décentralisées et autonomes, dont l'efficacité découle d'une coordination essentiellement numérique ${ }^{2}$. Il regroupe quantité de groupuscules franchisés sous une même bannière - la marque Tea Party - cherchant ainsi à se fédérer virtuellement autour d'un socle de valeurs commun. Ce processus rend la marque Tea Party tributaire de mécanismes d'autolégitimation fonctionnant selon un principe collaboratif semblable au Peer-to-Peer - la légitimation par les pairs - si l'on tient compte du caractère horizontal des échanges entre militants. Cette capacité à s'autoréguler (" self-police ») représente la réponse d'un mouvement décentralisé et sans hiérarchie pour palier au risque de déperdition de sens.

L'objectif du Tea Party consiste à faire émerger une nouvelle élite gouvernante qui s'engagerait à représenter davantage le peuple. Opposé à pénétrer l'arène politique de manière autonome, le Tea Party détermine ainsi les benchmarks (les points de références) qui conditionneront le choix du mouvement quant au soutien de candidats politiques « au parler-vrai », proches de leurs thèses. Cette forme de Peer-review (d'évaluation par les pairs) spécifique au consumérisme politique certifie les candidats comme aptes ou inaptes à jouir de l'aura de la marque. Dans le cas du Tea Party, ce soutien agit comme un label de qualité offert aux candidats, qui seront dès lors signalés comme politiquement vertueux.

Un fonctionnement hybride caractérise dès lors ce type de formation : plus proche d'un groupe d'intérêt que d'un parti classique, la résonnance offerte par les médias sociaux permet à ces mouvements d'inscrire leurs thèmes de prédilection au sein du débat démocratique

la fusillade meurtrière du 8 janvier 2010 à Tucson dans l'Arizona. Palin avait été à la base d'une campagne de diffamation (Smear Campaign) sur sa page Facebook qui visait, entre autre, l'élue démocrate Gabrielle Giffords, touchée lors de la fusillade.

1 Née en Nouvelle Angleterre au XVIIIe siècle, cette modalité politique renvoie à l'idée d'une démocratie directe ou de proximité dans laquelle les habitants se rassemblent dans une salle pour discuter ensemble, échanger librement leurs opinions et voter sur les questions concernant la communauté (Gallup, 2001).

2 On évoquera l'utilisation active de Facebook, Twitter ou YouTube destinée à l'organisation ou à la diffusion des vidéos des meetings. 
- comme agenda-setter alternatif - et de prendre part au processus de co-construction des marques politiques ${ }^{1}$.

\section{Le Movimento 5 Stelle}

À l'inverse de l'exemple précité, d'autres formations choisissentelles d'investir directement le système politique. Le MoVimento 5 Stelle ${ }^{2}$ de l'humoriste italien Beppe Grillo est ainsi entré dans l'arène politique en jouant les trouble-fêtes lors des élections régionales italiennes de mars 2010. Créé en octobre 2009, ce mouvement prônant la démocratie participative et directe se déclare en croisade contre « la caste » politique, contre Silvio Berlusconi et contre une gauche « désintéressée des problèmes des citoyens $»^{3}$.

La mobilisation des Grillini prend sa source dans la page Internet de Beppe Grillo, l'un des blogs les plus suivis au monde 4 et combine des actions off-line 5 à des initiatives en ligne. Une plateforme spécifique au mouvement fut ainsi lancée, s'appuyant sur le service web américain Meet $U p^{6}$ permettant aux militants de s'interconnecter et d'organiser des rencontres de terrain, donnant lieu à des actions contestataires mais également à l'établissement de listes civiques. Structuré en parti politique, le MoVimento 5 Stelle comptait fin 2011 une centaine de milliers d'inscrits et plusieurs représentants entrés dans différents conseils municipaux et régionaux. Le programme du parti se présente sous format numérique collaboratif : un Wiki permettant une amélioration en continu. Aussi, à l'instar du principe d'endossement des candidats par le Tea Party, le mouvement soutient les listes civiques en attribuant

1 Un type de procédé également expérimenté par Nicolas Hulot lors des présidentielles de 2007 en France. Déterminé à imposer l'environnement et la défense du climat au cœur de la campagne présidentielle, Hulot lança un livre-plateforme qui visait à interpeller les candidats et qui, s'il devait se lancer dans la course à l'Elysée, préfigurait son programme.

2 Les cinq étoiles représentent les points principaux du mouvement : l'eau publique, le « zéro déchets » (le tri, la réutilisation et le recyclage), les énergies renouvelables, la mobilité et le Wi-Fi libre et gratuit.

3 L'humoriste dénonce régulièrement les partis traditionnels comme « cancer de la démocratie ». Voir Beppe Grillo, le trublion qui chahute la gauche italienne, article paru dans Libération (2 avril 2010).

4 En 2011, le moteur de recherche Technorati classait le blog parmi les 20 premiers dans le monde.

5 Depuis 2007, un Vaffanculo Day (V-Day) est organisé par Grillo dans plusieurs centaines de villes italiennes, réunissant dizaines de milliers de personnes.

6 Disponible à : http://www.meetup.com/ 
à ces dernières un label de qualité (l'inscription officielle de la liste au parti).

\section{Le Parti pirate}

Mouvement d'opposition digital devenu formation politique, le Parti Pirate est apparu en 2006 en réaction à la loi du gouvernement suédois contre le partage de fichiers sur les réseaux Peer-to$P_{e e r^{1}}$. Constitué en Internationale depuis avril 2010 et présent dans une quarantaine de pays, le Parti Pirate regroupe aujourd'hui quantité de groupuscules franchisés sous un même étendard, respectant un programme similaire. Le parti se comprend comme une « formation spontanée née de la volonté des citoyens de se réapproprier une vie politique dans laquelle ils ne se reconnaissent plus » répondant à trois principes fondamentaux : la protection des droits (et données personnelles) des citoyens, la libération de la culture et du Wi-Fi - également au cour des revendications formulées par le MoVimento 5 Stelle - et la croyance que les brevets et les monopoles sont nuisibles au fonctionnement de notre société. Le Parti Pirate s'inscrit par conséquent dans le sillage d'un Internet militant marqué par une digitalisation des contenus et désirant « libérer les données publiques 》- un mouvement des « données ouvertes » (open data) sommant les administrations de mettre à la disposition du grand public les données brutes dont elles disposent (Cardon, 2010, p. 85).

Les trois formations précitées disposent de revendications communes : transparence du pouvoir, volonté d'inscrire l'activisme politique dans un projet participatif, e-Gouvernance pour plus de démocratie directe, etc. Ces mouvements politiques s'approprient les potentialités du Web 2.0 qui permettent désormais d'assurer une bidirectionnalité en politique : un feedback immédiat, voire une démocratie en temps réel. «La technologie, notamment, doit être utilisée dans le sens de la transparence et de l'immédiateté, et non pour ajouter à l'opacité et à la complexité » avise le programme du Parti Pirate. Il s'agit ici de s'extraire des principes de la civilisation du vote, dans lesquels nous sommes immergés, pour en envisager d'autres, esquissant par là une forme de démocratie de type post-représentatif (Hermet, Kazancigil \& Prudhomme, 2005, p. 121).

1 Une décision touchant principalement le site de partage Pirate Bay. 


\section{Du consumérisme politique au panoptique participatif}

Tandis que l'agrégation des citoyens-consommateurs engagés dans les mouvements contestataires auto-organisés susmentionnés marque l'extension du répertoire conventionnel de la participation, une évolution importante concerne le développement d'outils de surveillance et d'évaluation permanente des marques politiques. À la surveillance institutionnelle de l'État, autour de laquelle s'organise le débat sur les données personnelles précité, se superpose aujourd'hui une surveillance interpersonnelle d'un nouveau type (Bradwell \& Gallagher, 2007). À l'instar d'un Big Brother, tel qu'imaginé par George Orwell dans son roman 1984 ; grand surveillant omniprésent sur les « télécrans » des domiciles privés, la miniaturisation et la démocratisation de l'équipement hardware permettent une vigilance ascendante effectuée par pléthore de Little Brothers and Sisters. En dehors de tout principe coercitif, les citoyens-consommateurs s'inscrivent dans une dynamique de transparence et d'archivage holistique - un «panoptique participatif », d'après l'appellation formulée par le futurologue américain Jamais Cascio (2005) en référence au Panoptique imaginé par Jérémy Bentham au $18^{\text {ème }}$ siècle et décrit par Foucault (1975). Le panoptique participatif symbolise ici la somme d'une myriade de décisions rationnelles indépendantes prises par les individus; la version bottom-up d'une société sous écoute. Un regard médiaté exercé par les citoyens-consommateurs qui pourra également s'illustrer par la notion de « sousveillance » (Mann, Nolan \& Wellman, 2003).

Appliqué au consumérisme politique, cette dynamique ascendante débouche sur une monitory democracy telle qu'analysé par John Keane (2009) et décrivant, dans une situation de saturation médiatique, l'observation et le contrôle effectué par la société civile. Internet - comme forme politique - représente par conséquent l'expression réalisée de ces pouvoirs (Rosanvallon, 2006, p. 75). La mise en ligne instantanée de photos et de vidéos en corolaire à tout happening politique, alimentant en temps réel la grande conversation autours des marques, empêche en effet tout espace de " relâche » pour les élus.

Cette mise en conversation permanente exigera de la part des acteurs politiques un soin constant apporté à leur réputation digitale - un capital réputationnel qui revêt une importance décisive pour des marques politiques inscrites dans un espace virtuel critique et soumises au jugement continu des citoyens-consommateurs. 


\section{Conclusion}

L'autonomisation progressive de l'activisme politique débouche aujourd'hui sur des formes de mobilisation alternatives et sur l'avènement d'un espace public de type oppositionnel. Aussi, le mécontentement exprimé publiquement par ces mouvements peut se prévaloir d'une sorte de fonctionnalité démocratique (Mathieu, 2011, p. 154) susceptible d'aboutir à une synthèse entre initiatives contestataires et démocratie représentative. Il sera intéressant d'observer dans quelle mesure ces mobilisations numériquement boostées contribuent à contrecarrer le processus de désaffection politique, avec l'introduction d'une dimension participative horizontale comme remède à la crise de confiance " verticale ». Or, ce basculement vers le paradigme participatif ne concerne pour l'instant que les nouvelles formations politiques comme le Parti Pirate ou le MoVimento 5 Stelle, les partis traditionnels préférant miser sur l'efficacité organisationnelle des réseaux sociaux.

De manière plus critique, alors que le principe de « sous-veillance» évoque le risque d'une société de défiance généralisée (Lipovetsky, 2006, p. 363), le recentrage du politique sur les attentes des citoyensconsommateurs invite également à reconsidérer le statut de la représentativité classique et d'évaluer la possibilité d'une substitution progressive de la démocratie délégataire par une démocratie plébiscitaire, mieux adaptée au consumérisme politique.

\section{Références}

Blair, T. (1992). Pride without Prejudice. Fabian Review, 104(3), p. 3.

Bauwens, M., \& Sussan, R. (2005). Le peer to peer : nouvelle formation sociale, nouveau modèle civilisationnel, Revue du MAUSS, 26, pp. 193-210. doi :10.3917/ rdm.026.0193.

Blondeau, O. (2007). Devenir Média : L'activisme sur Internet, Entre défection et expérimentation. Paris : Éditions Amsterdam.

Blondiaux, L. (2008). Le nouvel esprit de la démocratie : actualité de la démocratie participative. Paris : Seuil et La République des Idées.

Bolz, N. (2002). Das konsumistische Manifest. Munich: Wilhelm Fink Verlag.

Boullier, D. (2008). La nouvelle matérialité de l'espace public : les dispositifs de la Netpolitique. Dans A. Mercier (Éd.), La communication politique, pp. 79-87. Paris : CNRS éditions.

Bradwell, P., \& Gallagher, N. (2007). We no Longer Control What Others Know About us, But We Don't Yet Understand the Consequences. Londres : Demos.

Bryman,A. (2002). The Disneyization of Society. Dans G. Ritzer(Éd.), McDonaldization: The Reader, pp. 52-59. Londres : Sage Publications.

Cardon, D. (2010). La démocratie Internet, Promesses et limites. Paris : Seuil et La 
république des Idées.

Cascio, J. (2005). The Rise of the Participatory Panopticon. Worldchanging: Bright Green. Disponible à : http://www.worldchanging.com/archives/002651.html

d'Almeida, N. (2007). La société du jugement. Essai sur les nouveaux pouvoirs de l'opinion. Paris : Armand Colin.

Ferrarese, E. (2007). Niklas Luhmann : une introduction. Paris : Pocket/La Découverte.

Foucault, M. (1975). Surveiller et punir. Paris : Gallimard.

Gallup, G. (2001). Sondage d'opinion et démocratie. Hermès, 31, pp. 167-180.

Hermet, G., Kazancigil, A., \& Prudhomme, J.-F. (2005). La gouvernance, un concept et ses applications. Paris : Karthala.

Hirschmann, A. (1995). Défection et Prise de parole, Paris : Fayard, 1995.

Keane, J. (2009). Monitory democracy and media-saturated societies. Griffith Review, pp. 1-23.

Lefebvre, R. (2008). L'opinion et la participation : la campagne présidentielle de Ségolène Royal. Hermès, 52, pp. 163-170.

Luhmann, N. (2000). Die Politik der Gesellschaft. Frankfut am Main: Suhrkamp.

Mann, S., Nolan, J., \& Wellman, B. (2003). Sousveillance: Inventing and Using Wearable Computing Devices for Data Collection in Surveillance Environments. Surveillance \& Society, 1(3), pp. 331-355. Disponible à http://www.surveillanceand-society.org/articles1(3)/sousveillance.pdf

Mathieu, L. (2011). La Démocratie protestataire: mouvements sociaux et politique en France aujourd'hui, Paris : Presse de Science Po.

Micheletti, M. (2003). Political Virtue and Shopping. Individuals, consumerism and collective action. New York: Palgrave MacMillan.

Negt, O. (2007). L'Espace public oppositionnel, Paris : Payot.

Nolte, P. (2011). Von der repräsentativen zur multiplen Demokratie, Aus Politik und Zeitgeschichte, 1(2), pp. 5-12.

Rosanvallon, P. (2006). La contre-démocratie. La politique à l'âge de la défiance. Paris : Seuil.

Séguéla, J., \& Saussez T. (2007). La prise de l'Elysée : Les campagnes présidentielles de la Ve République. Paris : Plon.

Trippi, J. (2005). The Revolution Will not Be Televised : Democracy, the internet, and the Overthrow of Everything. New York: Harper Collins.

Vedel, T. (2006). La révolution ne sera plus télévisée. Internet, information et démocratie. Pouvoirs, 119, pp. 41-54.

Vedel, T. (2003). L'idée de démocratie électronique. Origines, visions, questions. Dans P. Perrineau (Éd.), Le Désenchantement démocratique, pp. 243-266, La Tour d'Aigues : Éditions de l'Aube. 